\title{
CORRIGENDUM
}

\section{Experimental demonstration of a universally valid error-disturbance uncertainty relation in spin measurements}

Jacqueline Erhart, Stephan Sponar, Georg Sulyok, Gerald Badurek, Masanao Ozawa and Yuji Hasegawa

Nature Physics 8, 185-189 (2012); published online 15 January 2012; corrected after print 12 March 2012 and 1 August 2012.

In the version of this Letter originally published, in the Methods section under the heading 'Error and disturbance in spin measurements: theoretical determination' the equation defining $\eta(B)$ was incorrect - the term $\sigma_{\phi}$ should have been divided by 2 as shown below. This

$$
\eta(B)=\sqrt{2} \|\left[\sigma_{\phi} / 2, \sigma_{y}\right]|\psi\rangle \|=\sqrt{2} \cos \phi
$$

